\title{
Economical evaluation of large-scale photovoltaic systems using Universal Generating Function techniques
}

\author{
Yi DING (ه), Weixiang SHEN, Gregory LEVITIN, \\ Peng WANG, Lalit GOEL, Qiuwei WU
}

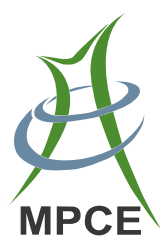

\begin{abstract}
Solar energy plays an important role in the global energy framework for future. Comparing with conventional generation systems using fossil fuels, the cost structure of photovoltaic (PV) systems is different: the capital cost is higher while the operation cost is negligible. Reliabilities of the PV system can also influence the cost for producing electricity. Investors, planners and regulators require deep insight into the return and cost of a PV project. A reliability based economical assessment of largescale PV systems has been conducted utilizing Universal Generating Function (UGF) techniques. The reliability models of solar panel arrays, PV inverters and energy production units (EPUs) are represented as the corresponding UGFs. The expected energy production models for different PV system configurations have also been developed. The expected unit cost of electricity has been calculated to provide informative metrics for making optimal decisions. The proposed method has been applied to determine the PV system configuration which provides electricity for a water purification process.
\end{abstract}

Keywords Photovoltaic system, Reliability, Economical assessment, Configuration

Received: 30 May 2012/ Accepted: 21 May 2013/Published online: 29 August 2013

(C) The Author(s) 2013. This article is published with open access at Springerlink.com

Y. DING, Q. WU, Department of Electrical Engineering,

Technical University of Denmark, Lyngby, Denmark

$(\bowtie)$ e-mail: yding@elektro.dtu.dk

W. SHEN, Faculty of Engineering \& Industrial Sciences, Swinburne University of Technology, Hawthorn, VIC, Australia

G. LEVITIN, Reliability \& Equipment Department, R\&D

Division, The Israel Electric Corporation Ltd., Haifa, Israel

P. WANG, L. GOEL, School of EEE, Nanyang Technological

University, Singapore, Singapore

\section{Introduction}

With the ever increasing concerns on environmental issues and the depletion of fossil fuels, the photovoltaic (PV) technology has drawn great attention and remarkable investments in the past decade [1]. This is due to the fact that the PV technology shows many advantages over other renewable energy technologies in terms of modularity, expandability, maintenance and reliability. In recent years, the contribution of the PV power generation to the grid has been rapidly increasing; at the current growth rate, it is expected to reach $2 \%$ of the world electricity generation by 2020 and up to $5 \%$ by $2030[2,3]$. During the next ten years, up to $15 \%$ of electricity in European Union will be produced by solar energy resources [4].

An important question for investors, planners and regulators is the return and cost of a PV project. The cost structure of PV systems is different from that of conventional generation system using fossil fuels such as coal, oil or natural gas. The initial capital cost is higher: basic components of a PV system-solar panels are quite expensive. However prices of solar panels are dropping fast: the average one-off installation cost of solar panels has already dropped from more than $\$ 2$ per unit of generating capacity in 2009 to about $\$ 1.50$ in 2011 [5]. On the other hand, there are no fuel cost and greenhouse gas emissions during the lifespan operation of 20-30 years. The maintenance cost of PV system is also relatively low.

PV systems are complicated engineering systems. A PV system is mainly composed of many solar panels and DC/ $\mathrm{AC}$ inverters. The trend of the fast growing PV systems is to adopt large-scale PV systems, which may require tens or hundreds of solar panels. Depending on input voltage ranges, maximum input currents and capacities of inverters, several solar panels are connected in series to form a 
string and a few strings are paralleled and tied up to a centralized-inverter or each of the strings is directly interfaced by a separate string-inverter or a combination of both, which are illustrated in Fig. 1. Different configurations have their own performance efficiencies for electricity production. When the performances of those configurations of PV systems are evaluated, it is assumed that the systems work without interruption. Although PV systems are relatively reliable, they may fail occasionally. Ignoring the effects of those failures may result in an optimistic estimation of energy production, which also decreases accuracy of cost assessment.

The approaches for improving the engineering system reliabilities are to increase the redundancy or/and reliability of the components in the system. For example, the use of multiple inverters in PV systems can increase system reliabilities. These approaches can improve the reliability of the PV systems and hence its energy production, but they may result in higher system cost. The reliability based cost assessment for renewable energy systems (RESs) and restructured generation systems has been studied in some recent research. Reference [6] provided a comprehensive analysis of the reliability and its cost implications on various choices of installation sites and operating policies as well as energy types, sizes and mixes in capacity expansion of the RESs. The genetic algorithm was used to optimize the offshore wind farms considering both energy production cost and system reliability [7]. A framework for analyzing the adequacy uncertainties of distributed generation systems was proposed in [8]. However reliability based economical evaluation of large-scale PV systems has not been comprehensively studied, which may be a useful analytical tool for assisting stakeholders in making optimal decision.
The large-scale PV system can be modeled as a typical multi state system (MSS). The UGF technique provides a systematic method for the performance and reliability assessment of MSS, which can replace extremely complicated combinational algorithms and reduce the computational burden [9-11]. Moreover, the UGF technique provides a flexible approach for representing reliability models of various energy systems. The UGF technique and genetic algorithm were used to determine the optimal structure of power systems subject to reliability constraints [12]. The reliability of flow transmission system was analyzed by using the combination of the UGF technique and extended block diagram methods [13]. The redundancy analysis of inter-connected generating systems was discussed in [14]. In [15], the UGF technique was used to determine the reserve expansion for maintaining the reliability level of power systems with high wind power penetration.

In this paper, the UGFs representing probabilistic performance distributions of solar panel arrays, PV inverters and energy production units (EPUs) are developed. The expected energy production models for PV systems under different configurations are also developed. The life cycle cost and annualized life cycle cost are evaluated to conduct economical assessment of a PV project. Moreover, a new economical index for PV systems-expected unit cost of electricity (EUCE) is developed for providing useful information.

Section 2 presents reliability models of large-scale PV systems. The developed UGFs are used to evaluate expected energy production. Cost analysis of PV systems is conducted in Section 3. Section 4 proposes a methodology to identify the feasible configurations of PV systems and determine the optimal one at the minimum EUCE. The

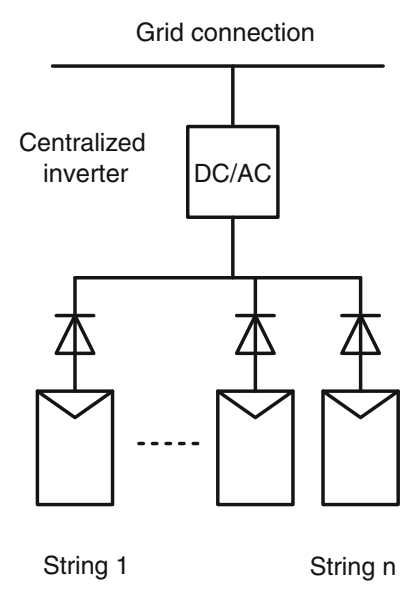

(a) Centralized inverter system

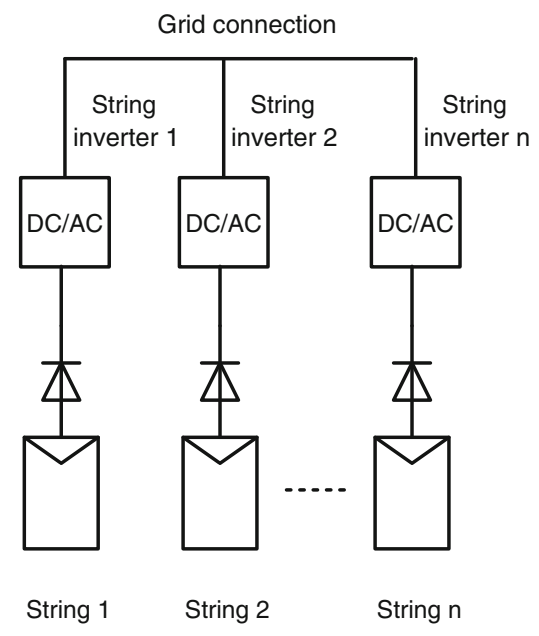

(b) String inverter system

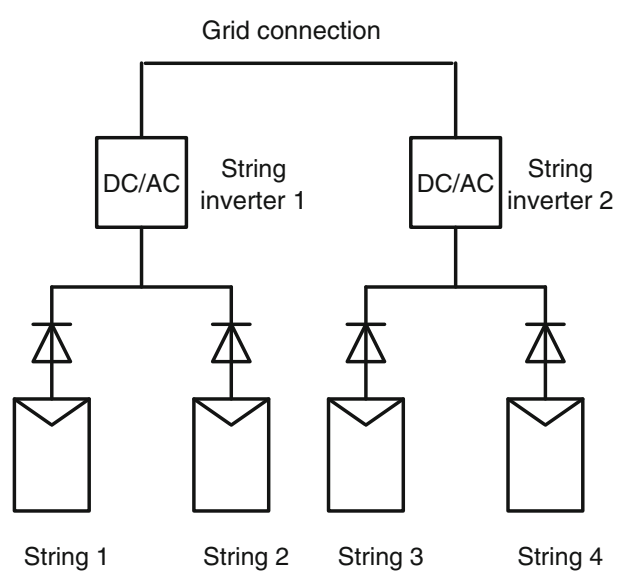

(c) Combination of centralized and string inverters

Fig. 1 Configurations of PV system 
proposed methods are used to assess the PV system configurations for providing electricity for a water purification process in Section 5. The conclusions are summarized in Section 6.

\section{Reliability models of PV systems}

A large-scale PV system basically consists of two major parts: solar panels and DC/AC inverters. Figure 2 shows a generalized configuration of the PV system. In the following, the reliability models of solar panel arrays, PV inverters and EPUs, and expected energy production calculation are discussed.

\subsection{Reliability models for solar panel arrays}

Solar panels are the key components of the PV systems. Solar panels can fail due to the degradation of mechanical properties of encapsulants, the adhesional strength, the presence of impurities, metalization, solder bond integrity and breakage, corrosion, and aging of backing layers, etc.

Given the failure rate $\lambda_{i}$ and repair rate $\mu_{i}$ of the solar panel $i$, the corresponding availability $A_{i}$ can be calculated by

$A_{i}=\mu_{i} /\left(\lambda_{i}+\mu_{i}\right)$

where $\lambda_{i}$ is the failure rate referring to the rate of departure from a component up-state (successful state) to its downstate (failure state) and $\mu_{i}$ is the repair rate referring to the rate of departure from the down-state to the up-state.

Some strings consist of several solar panels and a blocking diode in series. Any failure of a solar panel or a diode leads to the total failure of the string. Therefore, the availability of the string $s$ can be evaluated by:

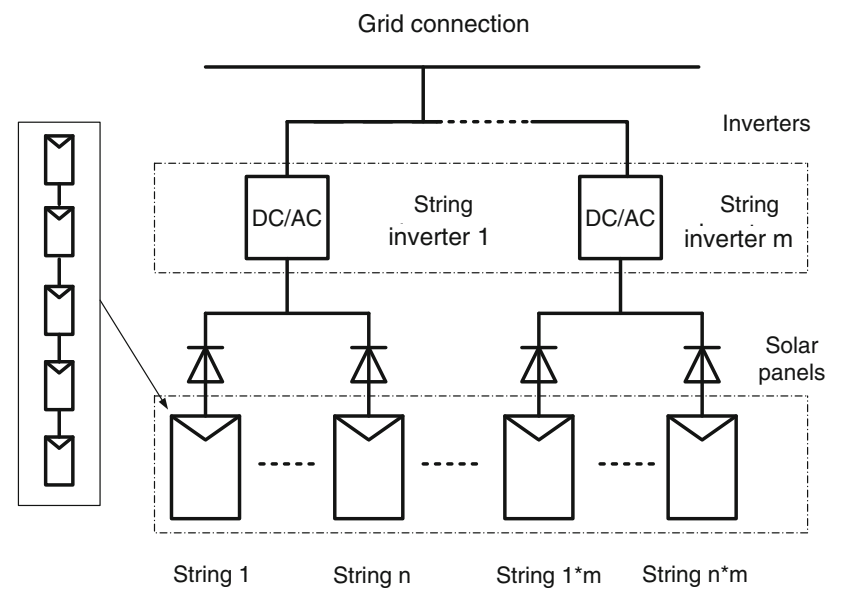

Fig. 2 Generalized configuration of PV system
$A_{s}=\prod_{i=1}^{n} A_{i} \cdot A_{d}$

where $n$ is a number of solar panels in the string and $A_{d}$ is the availability of the diode:

$A_{d}=\mu_{d} /\left(\lambda_{d}+\mu_{d}\right)$

where $\lambda_{d}$ and $\mu_{d}$ are the failure rate and the repair rate of the diode, respectively.

The available capacity $W_{s}$ of the string $s$ can be calculated as:

$W_{s}=\sum_{i=1}^{n} W_{i}-W_{d}$

where $W_{i}$ is the available capacity of the solar panel $i ; W_{d}$ is the capacity loss caused by the blocking diode, which can be determined as

$W_{d}=U_{d} \cdot I_{s}$

where $U_{d}$ is the voltage drop across the blocking diode and $I_{s}$ is the current of the string.

The UGF technique is proved to be very convenient for numerical realization and requires small computational resources [9-11] for performance and reliability evaluation of engineering systems [9]. Therefore, the UGF technique is used to evaluate the expected energy production of the PV system. The UGF representing the capacity distribution of a string $s$ can be defined as a polynomial:

$U_{s}(Z)=\sum_{k_{s}=1}^{2} p_{s, k_{s}} \cdot Z^{w_{s, k_{s}}}$

where $p_{s, k_{s}}$ and $w_{s, k_{s}}$ are the probability and the capacity level of state $k_{s}$ for the string $s, U_{s}(Z)$ represents the capacity distribution of the string $s, Z$ represents the $Z$-transform of any discrete random variables that has the probability mass function taking the form shown in (6) [10].

The string $s$ has two states: failure state and successful state. For the failure state, the capacity level and unavailability are 0 and $\left(1-A_{s}\right)$, respectively. For the working state, the capacity level and availability are $W_{s}$ and $A_{s}$, respectively.

A few strings are also arranged in parallel to form solar array and connected to a string inverter. Failure of any string in the array is tolerated without the loss of an entire array. However, the failure of a string degrades the available capacity of the array, leading to several de-rated states of the array. As a result, the solar array in the PV system can be regarded as a MSS. The parallel operator $\Omega_{\phi p}$ is applied for the parallel MSS by using associative and commutative properties. The parallel operator is a kind of composition operator to calculate the UGF for the parallel 
MSS, which strictly depends on the properties of the parallel structure function $[9,10]$. For example, if elements are connected in parallel, its capacity level for the state $k_{s}$ is the sum of the corresponding capacities $w_{s, k_{s}}(s=1,2, \ldots, N)$ of its elements, and the structure function for such a subsystem takes the form:

$\phi_{p}\left(w_{1, k_{1}}, w_{2, k_{2}}, \ldots, w_{N, k_{N}}\right)=\sum_{s=1}^{N} w_{s, k_{s}}$

The capacities of elements unambiguously determine the capacity of the subsystem or system. The transform, which maps the space of the element capacities into the space of the system capacity, is the system structure function $[9,10]$.

For a solar array with $N$ strings in parallel, its UGF can be obtained based on the UGFs for the arrays using the parallel composition operator $\Omega_{\phi p}$ over UGF representations of $N$ strings:

$$
\begin{aligned}
U_{a}(Z) & =\sum_{k_{a}=1}^{2^{N}} p_{a, k_{a}} \cdot Z^{w_{a, k_{a}}} \\
& =\Omega_{\phi p}\left(\sum_{k_{1}=1}^{2} p_{1, k_{1}} \cdot Z^{w_{1, k_{1}}}, p_{2, k_{2}} \cdot Z^{w_{2, k_{2}}}, \ldots, \sum_{k_{N}=1}^{2} p_{N, k_{N}} \cdot Z^{w_{N, k_{N}}}\right) \\
& =\sum_{k_{1}=1}^{2} \sum_{k_{2}=1}^{2} \ldots \sum_{k_{N}=1}^{2}\left(\prod_{s=1}^{N} p_{s, k_{s}} \cdot Z^{\sum_{s=1}^{N} w_{s, k_{s}}}\right)
\end{aligned}
$$

where $p_{a, k_{a}}$ and $w_{a, k_{a}}$ are the probability and the available capacity of the array in the state $k_{a}$, respectively. Equation (8) represents the capacity distribution of the solar array [8]: the coefficients of the terms in the polynomial (8) represent the probabilities of the array states while the exponents represent the corresponding capacities. The array has $2^{N}$ states.

\subsection{Reliability models for inverters}

PV inverters convert DC power from solar array into AC power, which matches the voltage of power grids. It is believed that inverters are the reliability bottleneck of PV systems and the vast majority of PV system failures are caused by inverters.

A typical PV inverter as shown in Fig. 3 includes four insulated-gate bipolar transistors (IGBTs) and an isolation transformer.

The failure rate of an IGBT is affected by the operating environment and other factors [16], which can be evaluated by

$\lambda_{I G B T}=0.5 \cdot \lambda_{b} \cdot \pi_{T} \cdot \pi_{Q} \cdot \pi_{E}$

where $\lambda_{b}$ is the base failure rate, $\pi_{T}, \pi_{Q}$ and $\pi_{E}$ are the temperature factor, the quality factor and the environment

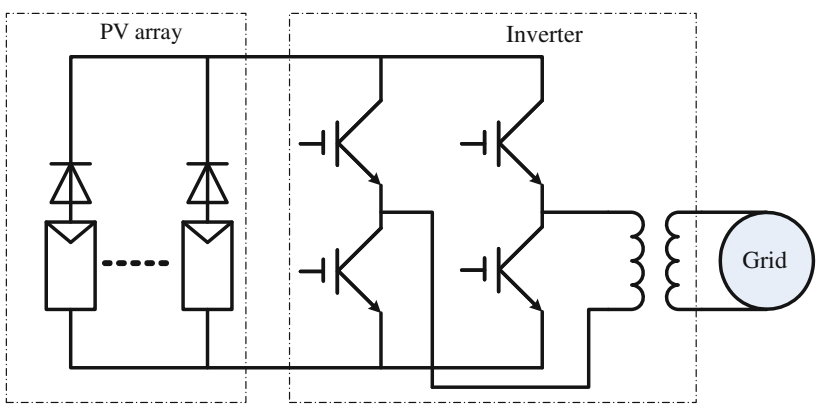

Fig. 3 Structure of inverter

factor, respectively. Therefore, the availability of the IGBT can be calculated as

$A_{I G B T}=\mu_{I G B T} /\left(\lambda_{I G B T}+\mu_{I G B T}\right)$

where $\mu_{I G B T}$ is the repair rate of the IGBT.

Similar equations can also be used to evaluate the availability $A_{\text {Trans }}$ of an isolation transformer. The components in a PV inverter are modeled as functional blocks connected in series. Any failure of an IGBT or an isolation transformer leads to the failure of an inverter. As a result, the availability of the PV inverter can be evaluated by

$A_{I}=\left(A_{I G B T}\right)^{m} \cdot A_{\text {Trans }}$

where $m$ is the number of IGBTs in the inverter. Therefore, the UGF representing the capacity distribution of the PV inverter is defined as

$U_{I}(Z)=\sum_{k_{I}=1}^{2} p_{I, k_{I}} \cdot Z^{w_{I, k_{I}}}$

where $p_{I, k_{I}}$ and $w_{I, k_{I}}$ are the probability and the capacity of state $k_{I}$ for the inverter.

The PV inverter has two states: failure state and successful state. For the failure state, the capacity level and the unavailability are 0 and $\left(1-A_{I}\right)$, respectively. For the successful state, the availability is $A_{I}$, and the capacity is determined by the nominal capacity of the inverter $w_{I, n}$ and the efficiency of the inverter $e_{I}$ :

$w_{I, 2_{I}}=w_{I, n} \cdot e_{I}$

\subsection{Reliability models for energy production unit}

A PV inverter is connected in series with the solar array to form an EPU. The series operator $\Omega_{\phi s}$ is used to calculate the UGF for an EPU using associative and commutative properties. For the type of the MSS containing elements connected in series, its capacity level for the state $k_{E}$ is the minimization of the corresponding capacities of its components. The structure function for an EPU takes the form: 
$\phi_{s}\left(w_{I, k_{I}}, w_{a, k_{a}}\right)=\min \left(w_{I, k_{I}}, w_{a, k_{a}}\right)$

The capacity distribution of the EPU $E$ can be obtained based on the UGF representing capacity distribution of the array and the PV inverter by using the series composition operator $\Omega_{\phi s}$ :

$$
\begin{aligned}
U_{E}(Z) & =\sum_{k_{E}=1}^{N_{E}} p_{E, k_{E}} \cdot Z^{w_{E, k_{E}}} \\
& =\Omega_{\phi s}\left(\sum_{k_{a}=1}^{2^{N}} p_{a, k_{a}} \cdot Z^{w_{a, k_{a}}}, \sum_{k_{I}=1}^{2} p_{I, k_{I}} \cdot Z^{w_{I, k_{I}}}\right) \\
& =\sum_{k_{a}=1}^{2^{N}} \sum_{k_{I}=1}^{2}\left(p_{a, k_{a}} \cdot p_{I, k_{I}} \cdot Z^{\min \left(w_{a, k_{a}}, w_{I, k_{I}}\right)}\right)
\end{aligned}
$$

where $N_{E}$ is the number of states of the EPU and equals to $2^{(N+1)}$.

\subsection{Expected energy production}

The PV system consists of several EPUs in parallel to supply electricity to the power grid. With the UGF for each EPU, the UGF representing the capacity distribution for the entire PV system can be calculated by using the parallel composition operator $\Omega_{\phi p}$ :

$$
\begin{aligned}
U_{s y s}(Z)= & \sum_{k=1}^{M} p_{k} \cdot Z^{w_{k}} \\
= & \Omega_{\phi p}\left(\sum_{k_{1}=1}^{N_{1}} p_{1, k_{1}} \cdot Z^{w_{1, k_{1}}}, \sum_{k_{2}=1}^{N_{2}} p_{2, k_{2}} \cdot Z^{w_{2, k_{2}}}, \ldots,\right. \\
& \left.\sum_{k_{E}=1}^{N_{E}} p_{E, k_{E}} \cdot Z^{w_{E, k_{E}}}, \ldots, \sum_{k_{m}=1}^{N_{m}} p_{m, k_{m}} \cdot Z^{w_{m, k_{m}}}\right) \\
= & \sum_{k_{1}=1}^{N_{1}} \sum_{k_{2}=1}^{N_{2}} \ldots \sum_{k_{m}=1}^{N_{m}}\left(\prod_{E=1}^{m} p_{E, k_{E}} \cdot Z^{\sum_{E=1}^{m} w_{E, k_{E}}}\right)
\end{aligned}
$$

where $m, M, p_{k}$ and $w_{k}$ are the number of EPUs, the state number of the PV system and the probability, and capacity level of state $k$ for the PV system, respectively.

The general technique for determining the UGF of the PV system is based on a state enumeration approach. This approach is usually extremely resource consuming. Fortunately, the PV system can be divided into subsystems (string, array and EPU) and the UGF method allows one to obtain the system UGF recursively. This property of the UGF method is based on the associative property of many practically used structure functions. The recursive approach presumes the UGF of subsystems containing several basic components and then treating the subsystem as a single component with the obtained UGF when the UGF of a higher level subsystem is computed [9]. The recursive approach provides a drastic reduction of the computational resources needed to obtain the capacity distribution of a complex MSS.

The yearly expected energy production (YEEP) of the $\mathrm{PV}$ system $E E_{p v}$ is defined as the product of the expected capacity of the system $E w$ and yearly peak sun hours $P S H$ :

$E E_{p v}=E w \times P S H$

The operator $\delta_{w}$ is used to calculate $E w$ and defined as

$E w=\delta_{w}\left(U_{s y s}\right)=\delta_{w}\left(\sum_{k=1}^{M} p_{k} \cdot Z^{w_{k}}\right)=\sum_{k=1}^{M} p_{k} \cdot w_{k}$

where $U_{s y s}$ is obtained from (16).

\section{Cost analyses of PV systems}

The cost of the PV system includes acquisition cost, operating and maintenance cost. These costs can be divided into two types. One is the recurring cost, e.g., operation and maintenance $(\mathrm{O} \& \mathrm{M})$ cost. The other is the initial capital cost, e.g., investment cost for purchasing solar panels and inverters. The life cycle cost (LCC) analysis evaluates the total system cost during the life span of the system. The LCC for all the parts in the system is added together to obtain the LCC for the entire system, where the system life cycle is assumed to be $T$ years. The LCC analysis converts the recurring cost into the present worth [17]. Annualized life cycle cost (ALCC) is also evaluated to provide an annualized "payment" required to fund the total system cost over the life span [18]. However LCC and ALCC analysis cannot evaluate the "equivalent" unit cost for producing electricity, which is important for determining the most cost-efficient system design. Therefore, a new economical index-EUCE is proposed to provide an informative metrics for evaluating cost efficiency of PV systems. The EUCE is defined as the system ALCC divided by YEEP.

LCC of the system is

$L C C=C_{\text {solar }}+C_{\text {inverter }}+C_{\text {om }}$

where $C_{\text {solar }}, C_{\text {inverter, }}$ and $C_{o m}$ are the costs of solar, inverter, and operation and maintenance (O\&M), respectively,

$C_{\text {solar }}=$ price of a solar panel $\times$ number of solar panel

$C_{\text {inverter }}=$ price of an inverter $\times$ number of inverters

$C_{o m}=C_{o m 0} \cdot P_{a 1}$

$P_{a 1}=X \cdot P_{a}$

$P_{a}=\left(1-X^{T}\right) /(1-X)$ 
$X=(1+i) /(1+d)$

where $P_{a}$ is the present worth, $X^{T}$ is the present worth factor for a cost in $T$ years, $i$ is the inflation rate, and $d$ is the interest rate [17].

Annualized life cycle cost (ALCC) is

$A L C C=L C C / P_{a}$

Expected unit cost of electricity (EUCE) is

$E U C E=A L C C / E E_{p v}$

\section{Feasible configuration identification for PV systems}

As shown in Fig. 2, a number of solar panels are connected in series to form a string, and a number of strings are paralleled and connected to a string inverter, then all the string inverters are connected to the power grid. Thus, in principle there are enormous configurations of the PV system for the given large number of solar panels. However, the feasible configurations are practically constrained by input voltage ranges, maximum input currents and capacities of the inverters. Consequently, only those configurations whose voltages, currents and capacities are within the normal operation range of the inverters will be considered. The constraints are specified as follows:

1) Input voltage limits

$V_{I}^{\min } \leq V_{s} \leq V_{I}^{\max }$

where $V_{s}$ is the voltage of the string connected to a string inverter, if the string consists of $n$ solar panels in series, then $V_{s}=n \cdot V_{p}, V_{p}$ is the voltage of the solar panel; $V_{I}^{\min }$ and $V_{I}^{\max }$ are the lower and upper limits, respectively.

2) Input current limit

$I_{I} \leq I_{I}^{\max }$

where $I_{I}$ is the input current of the string inverter, $I_{I}^{\max }$ is the maximum input current. If the array consists of $N$ strings in parallel, then $I_{I}=N \cdot I_{s}$, where $I_{s}$ is the current of the string.

3) Capacity limit
$W_{a} \leq W_{I}$

where $W_{a}$ is the available capacity of a solar array tied up to a string inverter, $W_{I}$ is the nominal capacity of the string inverter. $W_{a}=N \cdot n \cdot W_{p}$, where $W_{p}$ is the available capacity of the solar panel in the string. This constraint indicates that the available capacity of solar array tied up to a string inverter should be less than or equal to the nominal capacity of the inverter.

For various feasible configurations of the PV system, the economical efficiency is the major concern for comparing different design options. The reliability based cost analysis allows the investor or designer to evaluate the effects of different design options. A EUCE analysis discussed in the previous section can be helpful for determining the most cost-efficient system configuration.

The following three steps can be implemented for determining the most economically efficient design option:

1) Identifying all feasible configurations satisfying constraints (28)-(30). These identified configurations include the connections of solar panels, namely the number of solar panels in series and the number of strings in parallel, and the number of inverters required.

2) Evaluating the expected energy production and the EUCE of each configuration.

3) Determining the optimal configuration of the PV system at the minimum EUCE obtained from step 2).

\section{Application}

The proposed method is used to assess the performance and determine the feasible configurations of the PV system, which provides energy for a national demonstration project of water purification process in Singapore. According to electrical energy requirement of the process, it is estimated that 60 pieces of $175 \mathrm{Wp}$ (peak power) solar panels from SolarWorld are needed. For these 60 solar panels, all feasible configurations of the system are identified based on

Table 1 Parameters of inverters for evaluation of YEEP and system cost

\begin{tabular}{lllllll}
\hline Configuration & Capacity $(\mathrm{W})$ & Voltage $(\mathrm{V})$ & Current $(\mathrm{A})$ & Prices $(\mathrm{S} \$)$ & O \& M (S\$) & Peak efficiency $(\%)$ \\
\hline c12p12s05 & 1,100 & $139-400$ & 10 & 2,559 & 256 & 93.0 \\
c10p10s06 & 1,100 & $139-400$ & 10 & 2,559 & 256 & 93.0 \\
c06p06s10 & 2,500 & $224-600$ & 12 & 4,444 & 444 & 94.1 \\
c03p06s10 & 3,800 & $200-500$ & 20 & 5,083 & 508 & 95.6 \\
c02p06s10 & 5,500 & $246-600$ & 26 & 8,156 & 816 & 96.1 \\
c05p05s12 & 2,500 & $224-600$ & 12 & 4,444 & 444 & 94.1 \\
\hline
\end{tabular}

Note: one solar panel cost is $\mathrm{S} \$ 1,400$ and its monthly O\&M cost is assumed to $1 \%$ of solar panel cost, namely S\$14 each panel 


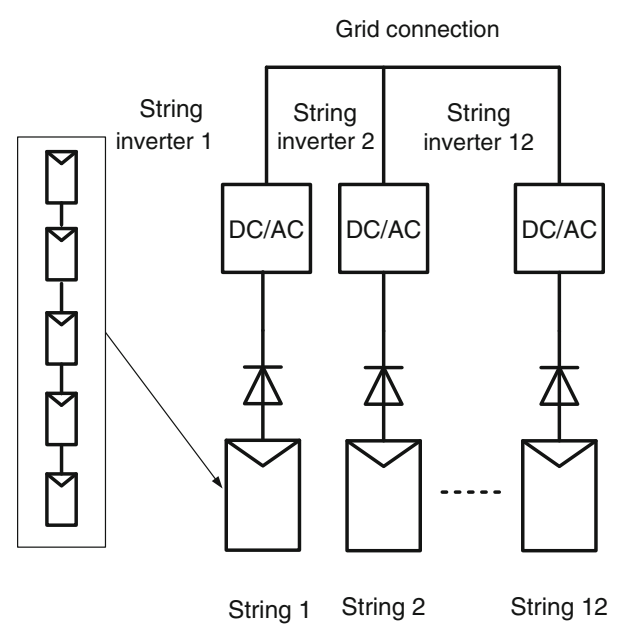

Fig. 4 Twelve string configurations with 12 inverters

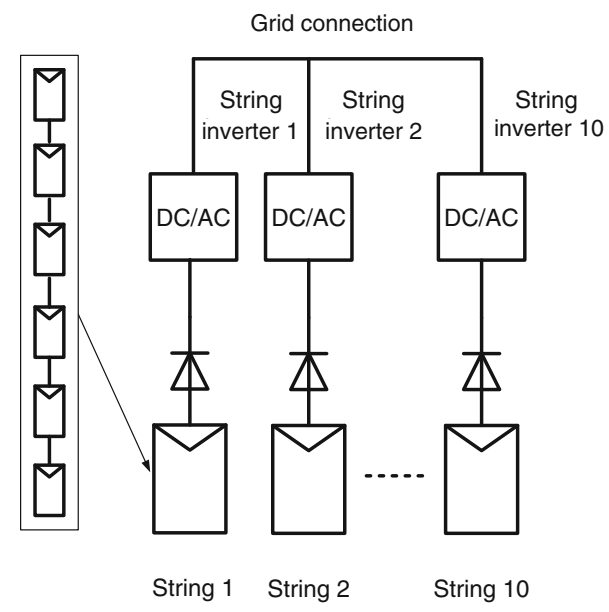

Fig. 5 Ten string configurations with 10 inverters the input voltage ranges, the maximum input currents and the nominal capacities of the inverters commercially available in the market, as shown in Table 1 and Figs. 4-7. Figure 4 shows one configuration of the system which consists of 12 strings each having 5 panels in series, Fig. 5 illustrates one configuration which consists of 10 strings each having 6 panels in series, Fig. 6 shows three configurations of the systems which consist of 6 strings each having 10 panels in series, and Fig. 7 shows one configuration of the system which consists of 5 strings each having 12 panels in series. It should be noted that the special "text string" is defined to represent each configuration, for example, the "c12p12s05" in Fig. 4 represents the configuration that includes $12 \mathrm{PV}$ inverters, 12 strings and each having 5 solar panels in series.

The basic parameters of the PV inverters including nominal capacity, input voltage range, purchasing cost, O\&M cost and peak efficiency for different configurations are shown in Table 1 [19].

For evaluating the failure rate of the IGBT of the inverter shown in Fig. 3 , the base failure rate $\lambda_{b}$ is set as 0.060 , the temperature factor $\pi_{T}$ is computed from $\pi_{T}=$ $\exp \left[-1,925\left(\frac{1}{T_{j}+273} \cdot \frac{1}{298}\right)\right]$ [20], where $T_{j}$ is the junction operating temperature of the device and set as $40{ }^{\circ} \mathrm{C}$, $\pi_{Q}=5.0$ and $\pi_{E}=1.0$ for the other conditions.

The repair rate of the IGBT equals to 0.0017 per hour. The failure rates of the solar panel and the string diode are set as 0.2068 and 0.0198 per million hours, respectively; the repair rates of the solar panel and the string diode are 4.0556 per year [20]. The current of the solar panel string is $4.89 \mathrm{~A}$ at the maximum power and the voltage drop of the blocking diode is set as $2.0 \mathrm{~V}$. The yearly peak sun hours

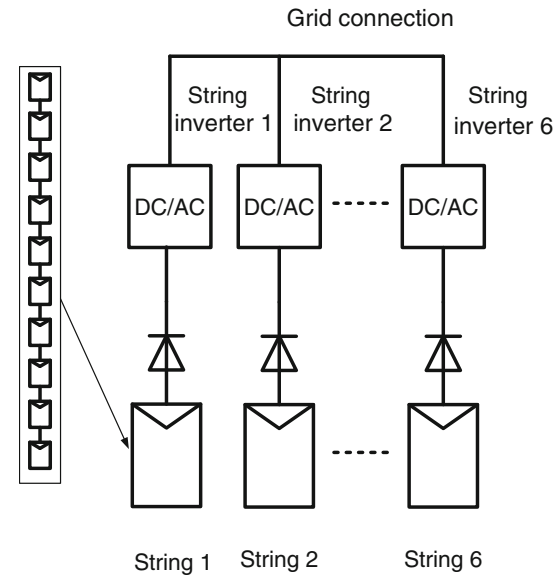

(a) $\mathrm{c} 06 \mathrm{p} 06 \mathrm{~s} 10$

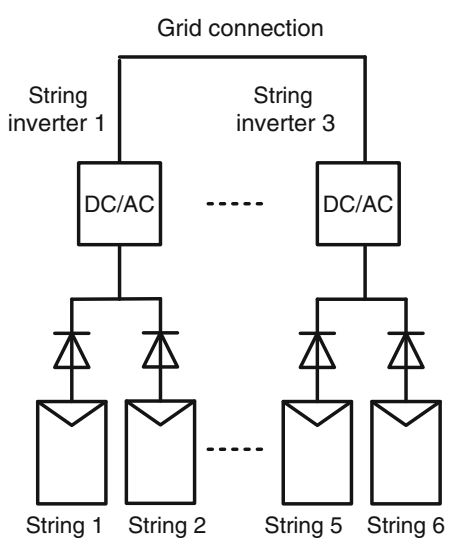

(b) $\mathrm{c} 03 \mathrm{p} 06 \mathrm{~s} 10$

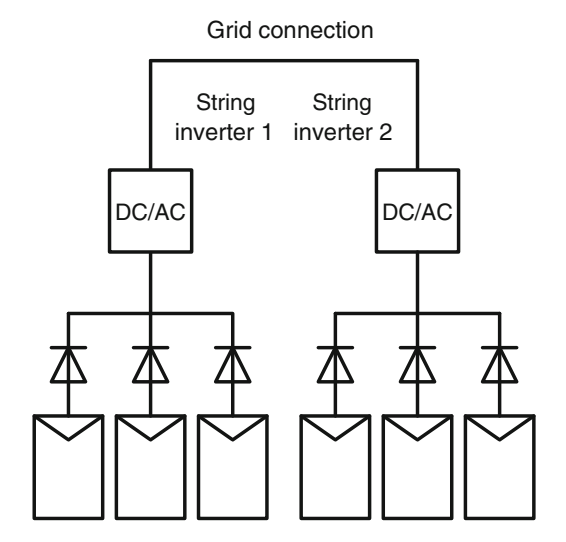

String 1 String 2 String 3 String 4 String 5 String 6 (c) $\mathrm{c} 02 \mathrm{p} 06 \mathrm{~s} 10$

Fig. 6 Six string configurations with different numbers of inverters 


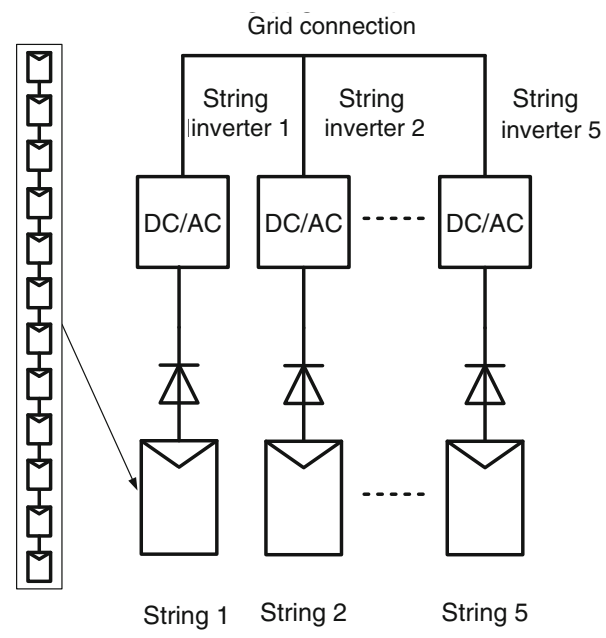

Fig. 7 Five string configuration with 5 inverters

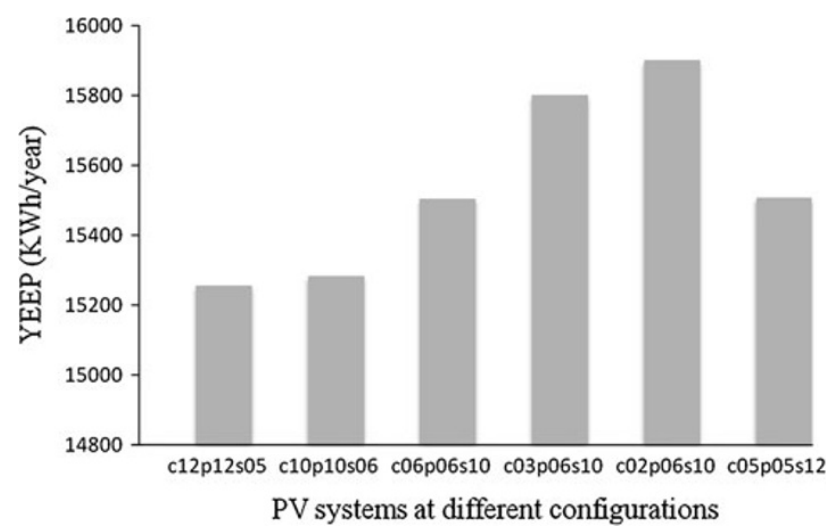

Fig. 8 Yearly expected energy production of PV systems

(PSHs) equals to 1,721.7 hours in Singapore, which is the average yearly PSHs over the period of 1993 to 2007 [21]. The inflation rate and the interest rate are assumed as $2.1 \%$ and $1 \%$, respectively. The system life cycle is assumed to be 20 years.

With all these parameters, the YEEP for each configuration is evaluated and the results are shown in Fig. 8. It can be observed from Fig. 8 that the configuration of "c02p06s 10 " has the highest expected energy production $(15,898.6 \mathrm{kWh} /$ year), which consists of 2 inverters, 6 strings, each of the strings having 10 solar panels in series. The YEEP for different configurations ranges from $15,255.7 \mathrm{kWh} /$ year to $15,898.6 \mathrm{kWh} /$ year, with the difference of $4.21 \%$. It represents that the difference of expected energy production for different configurations in the system life cycle can be $12,860 \mathrm{kWh}$.

The differences in the YEEP are mainly caused by the reliability differences of $\mathrm{PV}$ arrays and the connected PV

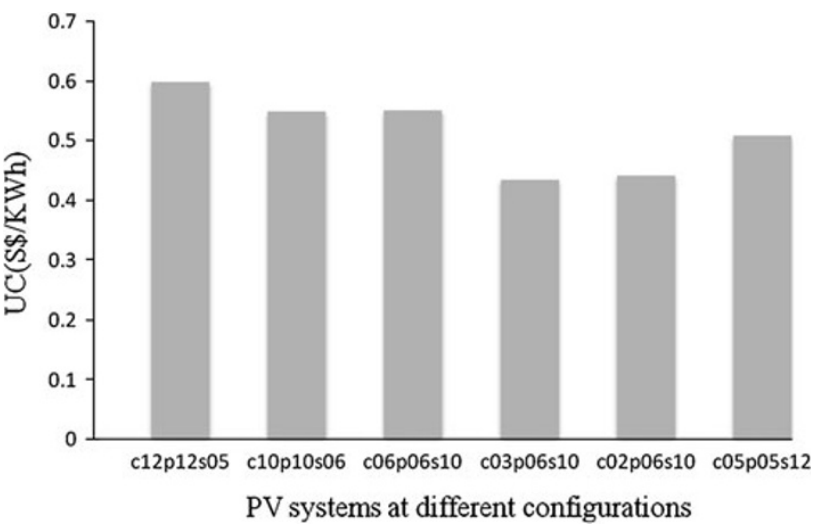

Fig. 9 Unit cost of PV systems

inverters for various configurations. The assessment of the ALCC for each configuration of the PV systems is also conducted. Consequently, the EUCE is easily calculated as the ratio of the ALCC to the YEEP, as shown in Fig. 9.

It can be seen that the EUCE for different configurations ranges from $0.434-0.598 \mathrm{~S} \$ / \mathrm{kWh}$. The configuration of "c03p06s10" has the lowest EUCE (0.434 S\$/kWh), which also has the second highest YEEP. The configuration of "c02p06s10" has the second lowest EUCE (0.441 $\mathrm{S} \$ / \mathrm{kWh}$ ) with the highest YEEP. The configuration of "c12p12s05" has the highest EUCE (0.598 S\$/kWh). Therefore, the "c03p06s10" is the optimal configuration with the lowest EUCE. The comparison results show that simply increasing the system cost by using relatively large number of low capacity inverters in the PV systems cannot guarantee high expected energy production, instead the configuration with high reliable PV arrays, and high capacity inverters can achieve the lowest unit cost of electricity.

\section{Conclusion}

In this paper, the UGF technique is used to represent reliability models of solar panel arrays, PV inverters and EPUs in a large-scale PV system. Based on the developed probabilistic performance distribution models, the expected energy production for PV systems is evaluated with respect to the reliability of system elements. The reliability based cost analysis of PV systems is conducted for providing informative metrics to stakeholders for making the optimal decision. A new economical index for PV systems-EUCE is also developed in this paper. The proposed method is used to identify the feasible configurations of PV systems and determine the economically optimal one. 
Open Access This article is distributed under the terms of the Creative Commons Attribution License which permits any use, distribution, and reproduction in any medium, provided the original author(s) and the source are credited.

\section{References}

[1] Hoffmann W (2006) PV on the way from a few lead markets to a world market. In: Conference Record of the IEEE 4th World Conference on Photovoltaic Energy Conversion, Vol 2, Waikoloa, HI, USA, 7-12 May 2006, pp 2454-2456

[2] IEA (2006) Trends in photovoltaic applications: survey report of selected IEA countries between 1992 and 2005. IEA-PVPS T115: 2006, The International Energy Agency, Paris, France

[3] IEA (2006) Chapter 6: Electricity. In: International Energy Outlook. Energy Information Administration, US Department of Energy, Washington, DC, USA

[4] Commission of the European Communities (2009) Communication from the commission to the European parliament, the council, the European Economic and Social Committee and the Committee of the Regions: investing in the development of low carbon technologies (SET-Plan). Commission Staff Working Document, Brussels, Belgium

[5] Clark D (2011) Price of solar panels to drop to $\$ 1$ by 2013. 2011-06-20. http://www.guardian.co.uk/environment/2011/jun/ 20/solar-panel-price-drop

[6] Rajesh K, Billinton R (2001) Reliability/Cost implications of PV and wind energy utilization in small isolated power systems. IEEE Trans Energ Conver 16(4):368-373

[7] Zhao M, Chen Z, Blaabjerg F (2009) Optimization of electrical system for offshore wind farms via genetic algorithm. IET Renew Power Gener 3(2):205-216

[8] Li YF, Zio E (2012) Uncertainty analysis of the adequacy assessment model of a distributed generation system. Renew Energ 41:235-244

[9] Lisnianski A, Levitin G (2003) Multi-state system reliability: assessment, optimization and applications. World Scientific, Singapore

[10] Levitin G (2005) Universal generating function and its applications. Springer, Berlin, Germany

[11] Ding Y, Lisnianski A (2008) Fuzzy universal generating functions for multi-state system reliability assessment. Fuzzy Set Syst 159(3):307-324

[12] Lisnianski A, Levitin G, Ben-Haim H et al (1996) Power system structure optimization subject to reliability constraints. Electr Power Syst Res 39:145-152

[13] Lisnianski A (2007) Extended block diagram method for a multi-state system reliability assessment. Reliab Eng Syst Safe 92(12):1601-1607

[14] Lisnianski A, Ding Y (2009) Redundancy analysis for repairable multi-state system by using combined stochastic processes methods and universal generating function technique. Reliab Eng Syst Safe 94(11):1788-1795

[15] Ding Y, Wang P, Goel L et al (2011) Long term reserve expansion of power systems with high wind power penetration using universal generating function methods. IEEE Trans Power Syst 26(2):766-774

[16] Military handbook: reliability prediction of electronic equipment. MIL-HDBK-217F, US Department of Defense, Washington, DC, USA

[17] Markvart T (2000) Solar electricity, 2nd edn. John Wiley \& Sons Inc, New York, USA

[18] Hoff J (2006) Equivalent uniform annual cost: a new approach to roof life cycle analysis. In: Proceedings of the RCI 21st
International Convention and Trade Show, Phoenix, AZ, USA, 23-38 Mar 2006, pp 115-124

[19] Solarzone. http://www.solazone.com.au/gridinvert.htm

[20] Copley controls. http://www.copleycontrols.com/Motion/pdf

[21] Solar energy engineering. http://energy.caeds.eng.uml.edu/

\section{Author Biographies}

Yi DING is an Associate Professor in the Department of Electrical Engineering, Technical University of Denmark (DTU), Denmark. He received the B.Eng. degree from Shanghai Jiao Tong University, China, and the Ph.D. degree from Nanyang Technological University, Singapore, both in electrical engineering. His research interests include power systems reliability/performance analysis incorporating renewable energy resources, smart grid performance analysis, and engineering systems reliability modeling and optimization.

Weixing SHEN received Ph.D. degree from the University of Hong Kong, Hong Kong, China, in 2002. Since 2009, he has been the Senior Lecturer in Electrical Engineering in the Faculty of Engineering and Industrial Sciences, Swinburne University of Technology, Australia. His research interests focus on electrical vehicles, renewable energy, power systems and power electronics. In these areas, he has published over 90 journal and conference papers.

Gregory LEVITIN received the M.S. degree in electrical engineering from Kharkov Polytechnic Institute, Ukraine, in 1982, the B.S. degree in mathematics from Kharkov State University in 1986, and the $\mathrm{Ph} . \mathrm{D}$. degree in industrial automation from Moscow Research Institute of Metalworking Machines in 1989. He is a Senior Expert at the Reliability Department of the Israel Electric Corporation, Haifa, Israel and Distinguished Visiting Professor of the Collaborative Autonomic Computing Laboratory at the School of Computer Science and Engineering, University of Electronics Science and Technology of China, Chengdu, China. He is the chair of the ESRA Technical Committee on System Reliability. He is an associate editor of IEEE Transactions on Reliability, area coordinator of International Journal of Performability Engineering, and member of the editorial boards of Reliability Engineering \& System Safety and Reliability and Quality Performance.

Peng WANG received the B.Sc. degree from Xi'an Jiaotong University, Xi'an, China, in 1978, the M.Sc. degree from Taiyuan University of Technology, Taiyuan, China, in 1987, and the M.Sc. and $\mathrm{Ph} . \mathrm{D}$. degrees from University of Saskatchewan, Saskatoon, SK, Canada, in 1995 and 1998, respectively. Currently, he is an Associate Professor with the School of Electrical and Electronic Engineering, Nanyang Technological University, Singapore.

Lalit GOEL was born in New Delhi, India, in 1960. He obtained his B. Tech. degree in electrical engineering from the Regional Engineering College, Warangal, India, in 1983, and his M.Sc. and Ph.D. degrees in electrical engineering from the University of Saskatchewan, Canada, in 1988 and 1991, respectively. He joined the School of EEE at the Nanyang Technological University (NTU), Singapore, in 1991 where he is presently a professor. He served as the Head of the Division of Power Engineering from July 2005 to August 2008. From July 2008 until June 2012 he served as the Dean of Admissions \& Financial Aid. He is currently appointed as Director, Undergraduate Education (Projects) in the President's Office, NTU. He is a Fellow of the IEEE. His research interests are power system reliability, cost/benefit assessment, power markets and renewables. 
Qiuwei WU obtained the B. Eng. and M. Eng. from Nanjing University of Science and Technology, Nanjing, P. R. China, in 2000 and 2003, respectively, both in Power System and Automation. He obtained the Ph.D. degree from Nanyang Technological University, Singapore, in 2009 in Power System Engineering. He has been an assistant professor with CET since November 2010. His research interests are integration of electrical vehicles (EVs) into power systems, integration study for wind power, dynamic performance of power systems, real time simulation of power systems using RTDS and reliability analysis and improvement of restructured power systems using demand response programs. 